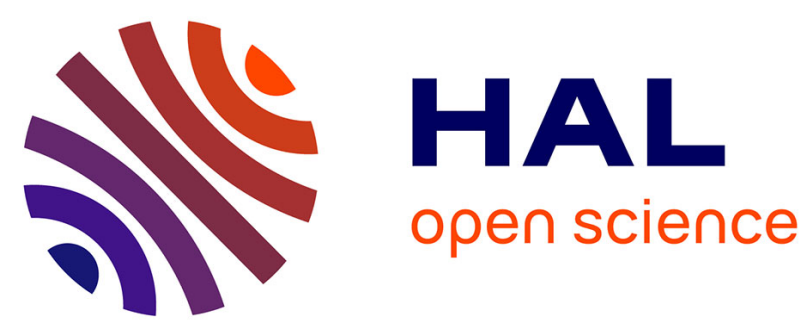

\title{
La conception innovante comme mode d'extension et de régénération de la conception réglée: les expériences oubliées aux origines des bureaux d'études.
}

Pascal Le Masson, Benoit Weil

\section{- To cite this version:}

Pascal Le Masson, Benoit Weil. La conception innovante comme mode d'extension et de régénération de la conception réglée: les expériences oubliées aux origines des bureaux d'études.. Entreprises et Histoire, 2010, 58, pp.51-73. hal-00696132

\section{HAL Id: hal-00696132}

https://hal-mines-paristech.archives-ouvertes.fr/hal-00696132

Submitted on 10 May 2012

HAL is a multi-disciplinary open access archive for the deposit and dissemination of scientific research documents, whether they are published or not. The documents may come from teaching and research institutions in France or abroad, or from public or private research centers.
L'archive ouverte pluridisciplinaire HAL, est destinée au dépôt et à la diffusion de documents scientifiques de niveau recherche, publiés ou non, émanant des établissements d'enseignement et de recherche français ou étrangers, des laboratoires publics ou privés. 


\title{
LA CONCEPTION INNOVANTE COMME MODE D'EXTENSION ET DE REGENERATION DE LA CONCEPTION REGLEE : LES EXPERIENCES OUBLIEES AUX ORIGINES DES BUREAUX D'ETUDES
}

\author{
Pascal Le Masson, et Benoit Weil, Centre de Gestion Scientifique, MINESParisTech ${ }^{1}$
}

Abstract :

Les Bureaux d'Etudes sont l'objet de critiques pour leur faible capacité à innover et gérer le renouvellement des objets qu'ils conçoivent et des compétences associées. Pourtant l'examen de la genèse au XIXème siècle des premiers Bureaux d'Etudes fait apparaître des capacités de conception plus riches. A côté de la conception réglée il s'y déployait des formes originales de conception innovante qui assuraient la régénération régulière des règles, et qui étendaient l'espace des conceptions atteignables en augmentant la valeur ou en ouvrant de nouvelles lignées de produits. Deux exemples tirés de l'histoire de Gustave Eiffel et de son Bureau d'Etudes le mettent en évidence, ouvrant des pistes pour penser la dynamique des Bureaux d'Etudes aujourd'hui.

\section{Introduction}

L'émergence et le développement des Bureaux d'Etudes à la fin du XIXème siècle apparaissent comme une étape charnière dans la constitution de la grande entreprise industrielle. Ce mouvement se développe progressivement aux Etats Unis [1] [2] et en Allemagne [3] mais également en France et dans d'autres pays. Il s'agit d'un effort de domestication de la conception qui passe par la structuration et l'organisation de la conception réglée, i.e. une conception construite sur l'utilisation collective efficace d'un système de règles stables permettant renouvellement, amélioration et croissance de la variété des produits (Le Masson et Weil, 2008). Cette forme d'organisation permet aux entreprises qui l'adoptent d'atteindre des performances remarquables. Elle connaît alors une diffusion rapide au point d'éclipser les formes d'organisation alternatives existant jusqu'alors (en particulier la figure de l'entrepreneur-innovateur) et d'apparaître bientôt comme incontournable dans la grande entreprise industrielle [4].

Pourtant on n'a généralement retenu du Bureau d'Etudes qu'une partie de sa logique, et d'autres expériences imaginées et conduites dans cette période de gestation, n'ont pas connu la même postérité. A partir de l'examen de l'une de ces expériences remarquables, mais oubliées, nous voudrions montrer comment les premiers promoteurs des Bureaux d'Etudes avaient tentés de concilier deux logiques de conception et de combiner une double organisation l'une, visant la conception réglée, un système de règle permettant la domestication de la conception, l'autre, organisant collectivement l'innovation afin de

\footnotetext{
${ }^{1}$ Cette recherche et cette publication ont bénéficié du soutien du programme ANR RITE (R.\&D., Innovation et Transformation des Entreprises : apports et limites d'une théorie des régimes de conception) (ANR-07ENTR-011-RITE) et de la chaire « Théorie et Méthodes de la Conception Innovante » de MINES ParisTech.
} 
régénérer régulièrement le système de règles et/ou d'explorer et d'étendre la valeur en détournant les règles en vigueur dans le Bureau d'Etudes. Si la première est bien connue et largement diffusée en pratique, la seconde, rarement mise en avant, n'a pas donné lieu à une doctrine spécifique et la pratique l'a largement délaissé. Or ces deux volets de l'organisation interagissaient fortement et se complétaient utilement, la seconde conditionnant même les performances de la première.

Nous commencerons par présenter les caractéristiques principales de ces organisations collectives de l'innovation en les confrontant à celles des Bureaux d'Etudes. Nous proposerons ensuite un cadre d'analyse mobilisant les théories les plus récentes de la conception. Celui-ci nous permettra de distinguer ces différentes formes et logiques d'organisation et de repérer leurs interactions dans les deux exemples historiques retenus basés sur l'expérience d'Eiffel. Pour finir nous nous interrogerons sur les raisons de cette étrange amnésie qui ne nous a conservé des Bureaux d'Etudes qu'une perspective mutilée. Or retrouver cette histoire et en proposer une doctrine peut s'avérer un enjeu majeur pour éclairer les mutations contemporaines des régimes de conception et aider à l'émergence de formes nouvelles d'organisation de la conception.

\section{1- Bureaux d'Etudes et conception réglée : un modèle incomplet?}

Depuis la fin du XIX ème siècle les Bureaux d'Etudes sont associés à l'organisation de la conception dans la grande entreprise industrielle. Ce mode d'organisation emblématique semble aujourd'hui bien connu, il incarne de façon archétypale la logique de la conception réglée. Le modèle du Bureau d'Etude tel qu'on l'a retenu historiquement repose sur trois propriétés principales :

- Un raisonnement de conception basé sur l'application d'un système de règles. Ce système de règles assure aux concepteurs qu'ils pourront concevoir une variété d'objets d'un certain type sans se lancer dans de coûteuses et incertaines explorations. Pour un "modèle objet» donné les concepteurs disposent d'un «modèle génératif» qui propose un chemin de conception et organise l'enchaînement des étapes à parcourir et de «modèles conceptuels » qui relient par des règles les différentes variables de description du «modèle objet». Le passage du cahier des charges à la proposition d'une solution s'organise suivant une logique de programmation et de problem solving.

- une logique d'organisation de type bureaucratie, liée à une structuration des connaissances (le système de règles). Cette organisation rend possible la constitution de métiers et la professionnalisation en lien avec les écoles techniques et les écoles d'ingénieurs. Elle permet également la division du travail de conception au sein d'un secteur industriel stabilisé.

- Une logique de croissance liée à une économie de la production de connaissances, à la réduction des coûts et des incertitudes. Le pouvoir d'expansion est limité à la variété atteignable dans le cadre d'un modèle d'objet stabilisé. Mais comme le Bureau d'Etudes peut atteindre tous ces points en réutilisant au maximum les connaissances existantes (dans le système de règles) et en minimisant les coûts de production de connaissances nouvelles, le rendement de la conception est remarquable.

Cette forme d'organisation de la conception réglée présente de nombreux avantages. Elle combine une forte expansion (variété des produits conçus) et une relativement faible consommation des ressources. Elle oriente le développement de compétences (et 
leurs diffusions) parfaitement adaptées au modèle objet à concevoir, en particulier elle favorise le développement de laboratoires de recherche qui se verront assignés des questions bien formulées. Elle prescrit et organise un système industriel de production de masse en tirant parti de tous les effets de rendement possibles. Elle favorise l'instauration de secteurs industriels dans le cadre de relations de marché qu'elle permet de structurer. Bref certains auteurs considèrent même que l'apparition des Bureaux d'Etudes était un maillon indispensable de la constitution des grandes entreprises industrielles [5-7].

Des critiques n'ont pourtant pas non plus manquées d'être formulées à l'encontre de cet archétype des Bureaux d'Etudes. Il enfermerait l'entreprise dans le cadre étroit d'un dominant design stabilisé dont elle aurait ensuite les plus grandes difficultés à sortir [8]. Les innovations ne porteraient généralement que sur des paramètres de performance déjà bien identifiés du modèle d'objet [9]. Les innovations de ruptures resteraient rares et aléatoires [10]. La révision ou la régénération du système de règles apparaîtrait souvent extrêmement difficile alors même qu'elles semblent indispensables [11]. L'exploration de la valeur, de nouveaux business model voire l'ouverture de nouveaux marchés demeureraient hors du champ atteignable par cette forme d'organisation.

Ces critiques s'expriment aujourd'hui avec d'autant plus de virulence que l'époque actuelle appelle une forme d'innovation intensive beaucoup plus marquée qui disqualifierait le bureau d'études et la conception réglée.

Mais ne sommes nous pas victime d'une erreur de perspective? L'archétype du Bureau d'Etudes tel qu'il est communément admis et tel que nous venons de le présenter correspond-il effectivement à la richesse des formes d'organisation expérimentées à la fin du XIXème siècle ?

Nos doutes sont renforcés par un ensemble d'éléments historiques bien connus qui laissent penser que cet archétype ne rend compte que d'une partie de ce qui était possible et de ce qui a été expérimenté.

- les logiques de croissance à la fin du XIX et au début du XX ne se contentaient pas de poursuivre des économies d'échelle et minimiser les ressources consommées, elles cherchaient aussi à étendre l'espace de la valeur de ce qui était conçu (produits, services,...) ; ce dont témoigne l'histoire économique de cette période avec l'apparition et le développement, par exemple de l'avion, du téléphone, de l'automobile, de l'éclairage électrique, de la chimie,...

- La régénération du système de règles du Bureau d'Etudes apparaît fréquemment. On n'assiste non seulement à leur sophistication mais surtout à la floraison de systèmes de règles variés (génie électrique, thermodynamique, mécanique des structures,...) qui accompagnent l'intégration des nouvelles techniques et l'extension des espaces fonctionnels couverts par les produits de cette époque.

- Les organisations en place ne sont pas uniquement des bureaucraties et prennent souvent des formes plus complexes. Ainsi les organigrammes de Thomson Houston en 1888 font apparaître à côté d'un Bureau d'Etudes (engineering department) implanté à l'usine une seconde entité vouée à l'invention (model room) où s'activent Elihu Thomson et son équipe[12].

L'explication classique généralement avancée est la coexistence et la compétition de deux systèmes. Certains auteurs [12,13] [14] décrivent une logique d'entrepreneur 
innovateur, rapidement remplacée par une bureaucratie (BE). D'autres auteurs défendent la thèse d'une forme d'ambidextrie industrielle [15].

Nous voudrions dans ce papier explorer une autre hypothèse : l'archétype du Bureau d'Etudes ne correspond qu'à l'un des aspects des formes d'organisation qui se sont inventées à la fin du XIXème siècle. Au moins dans un certain nombre de cas, un autre volet visait à l'organisation collective de la conception innovante à côté et en lien avec la conception réglée qui seule a survécue, et seule a donné lieu à une doctrine.

Nous pouvons alors formuler les deux questions de recherche auxquelles nous souhaitons répondre dans ce papier.

\section{1) Existait-il des formes d'organisation collective de la conception innovante dans les firmes qui les premières ont tenté d'organiser des Bureaux d'Etudes?}

A ces organisations de la Conception Innovante seraient associés les trois traits caractéristiques suivants :

- un raisonnement de conception qui viserait la régénération des langages de description de l'objet (à la fois le système de règles et le modèle objet lui-même)

- une organisation collective complexe qui ne se décrit pas dans les termes d'une bureaucratie et s'apparente à un métabolisme [7].

- une logique de croissance s'appuyant sur une expansion de l'espace des objets conçus, croissance par l'apparition de nouvelles identités (nouvelles lignées de produits) ou par l'adjonction de nouvelles dimensions ou de nouvelles valeurs à des familles d'objets déjà connus.

\section{2) Comment ces formes d'organisation collective de la Conception Innovante s'articulaient-elles avec la Conception Réglée ?}

Cette articulation devrait se manifester à chacun des trois niveaux de description que nous utilisons :

1) Sur le raisonnement: la Conception Innovante a pour mission la régénération du système de règles, mais dans l'autre sens profite aussi du Bureau d'Etudes et du système de règles existant.

2) Sur l'organisation: le métabolisme de la Conception Innovante favorise la transformation des métiers de la Conception Réglée soit par l'émergence et la constitution de nouveaux métiers, soit par adjonction de nouvelles compétences aux métiers existants.

3) Sur les logiques de croissance : la Conception Innovante explore de nouveaux espaces de valeurs et propose de nouvelles "têtes de lignées » qui pourront ensuite être exploitées par la Conception Réglée ; a contrario la Conception Réglée peut désigner des espaces de tensions qui méritent un effort d'expansion conceptuel.

2- Méthodologie et cadre d'analyse : distinguer "conception réglée " et " conception innovante " et repérer leurs articulations

Ce papier ne prétend pas apporter une réponse définitive aux deux questions précédentes, il s'agit davantage d'une recherche exploratoire. Il vise à proposer de 
premiers éléments allant dans le sens des hypothèses formulées. Elle appelle des investigations plus nombreuses pour confirmer ces premiers résultats.

La méthodologie de recherche utilisée repose sur l'étude de cas remarquables où une entreprise combine un Bureau d'Etudes et des capacités d'organisation collective de la conception innovante. La difficulté tient à la capacité à distinguer des activités et des organisations qui relèvent de la conception réglée de celle que l'on peut associer à la conception innovante et à repérer leurs articulations. Pour cela nous avons construit à partir des formalismes les plus récents du raisonnement de conception un cadre d'analyse qui nous permet de suivre les raisonnements de conception et de révéler les formes de conception dans les organisations étudiées.

Nous avons retenu la théorie C-K [16] [17] pour fabriquer notre instrument d'analyse, car parmi les théories de la conception, elle est celle qui permet de rendre compte dans un même formalisme, tout en les distinguant, des situations de conception réglée et de conception innovante.

La théorie $\mathrm{C}-\mathrm{K}$ modélise le raisonnement de conception comme l'interaction de deux espaces, l'espace des concepts (C) et l'espace des connaissances (K). Si les propositions dans l'espace $\mathrm{K}$ ont un statut logique, elles sont soit vraies soit fausses, celles dans $\mathrm{C}$, les concepts, n'en ont pas, elles sont indécidables. Un concept, point de départ de la conception, est donc une proposition formée à partir de termes qui ont un sens dans l'espace des connaissances mais qui n'est ni vraie ni fausse (on parlera de disjonction). Pour avancer dans la conception (voir figure 1) il s'agira d'ajouter progressivement des attributs au concepts initial (ces attributs ne peuvent provenir que de $\mathrm{K}$ ). On opère ainsi des partitions, celles-ci peuvent être restrictives ou expansives (si elle modifie le modèle d'objet préexistant dans l'espace $\mathrm{K}$ ). Le processus de conception mobilise 4 opérateurs de $\mathrm{K}$ dans $\mathrm{K}$, de $\mathrm{C}$ dans $\mathrm{K}$, de $\mathrm{C}$ dans $\mathrm{C}$ et de $\mathrm{K}$ dans $\mathrm{C}$. Le raisonnement de conception consiste ainsi à mener conjointement une double expansion de l'espace $\mathrm{C}$ et de l'espace $\mathrm{K}$. La conception s'arrête quand le concept acquiert un statut logique dans $\mathrm{K}$ (il soit vrai soit faux).

Figure 1 : Théorie C-K (source : Hatchuel and Weil, 2009)

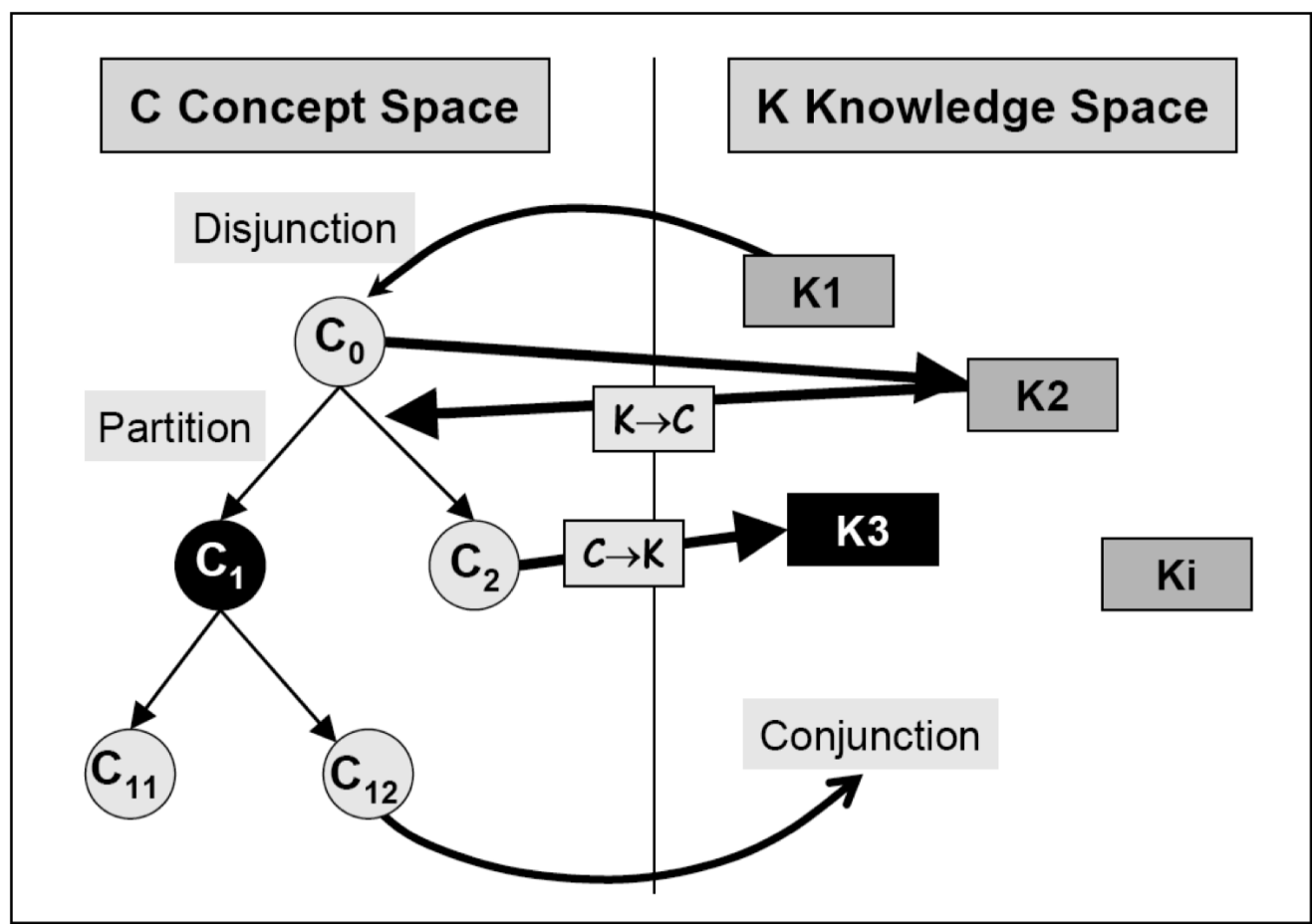


En utilisant ce formalisme nous pouvons schématiser et contraster les traits caractéristiques des situations de conception réglée et de conception innovante (voir figure 2).

En conception réglée : Les connaissances correspondent au système de règles, il est distribué dans des poches de connaissances associées à des acteurs spécialisés. Dans l'espace $\mathrm{C}$ le raisonnement de conception s'appuyant sur ce système de règles suit un chemin prédéterminé par le Modèle Génératif et décrit progressivement les différents paramètres du Modèle Objet. L'expansion en $\mathrm{C}$ se limite à explorer la variété associée à un Modèle Objet, nous l'avons représenté par un cône au dernier niveau de notre arborescence. L'expansion en K est aussi limitée que possible pour limiter les coûts de production des connaissances. Le systèmes de règle n'évolue donc que marginalement au cours de la conception. Nous retrouvons les trois traits principaux que nous avons mentionnés dans la première partie.
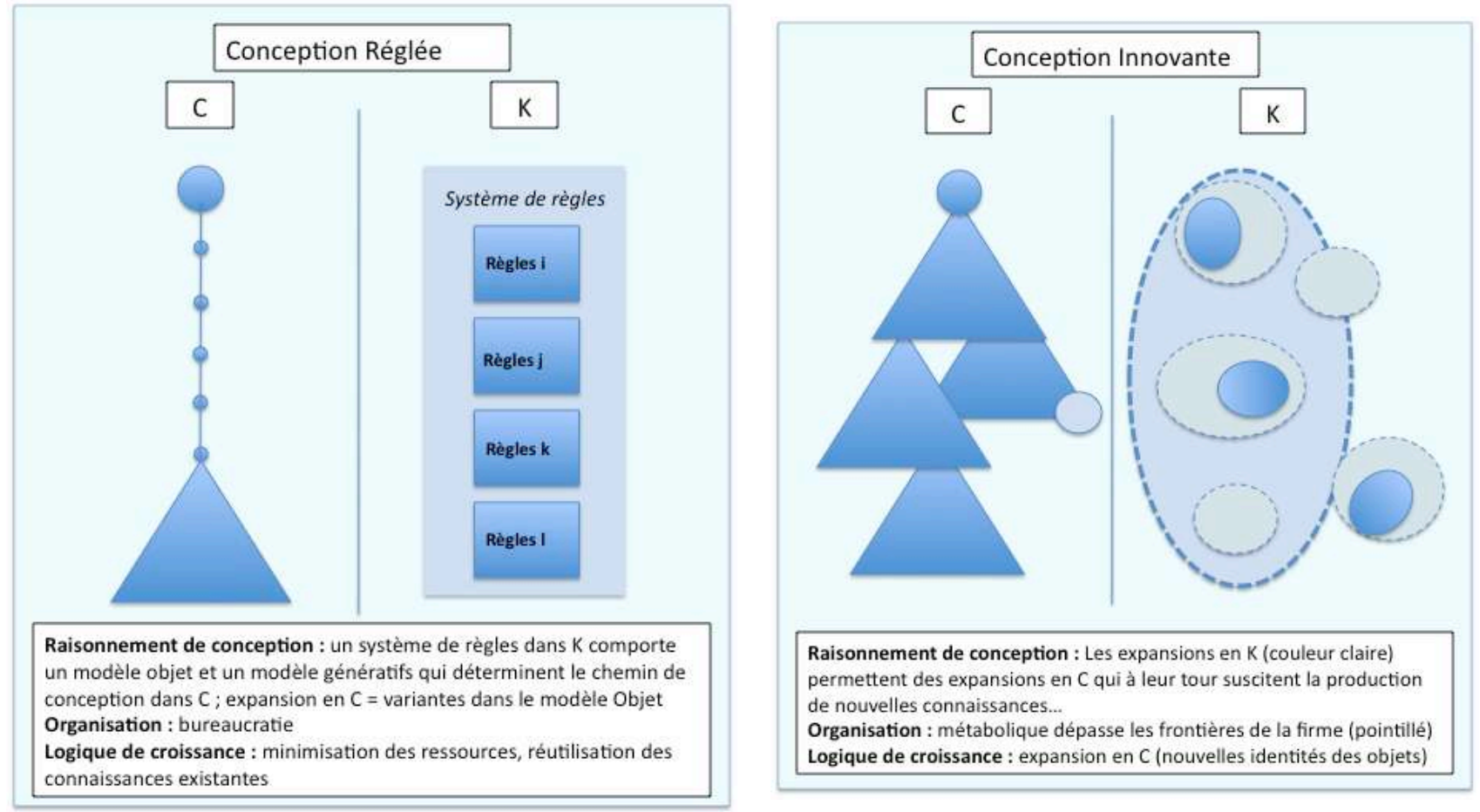

Figure 2 : Traits stylisés de la Conception Réglée et de la Conception Innovante

En conception innovante : l'exploration en $\mathrm{C}$ est beaucoup plus ouverte à tous les niveaux, elle part d'un concept i.e. une proposition indécidable dans l'espace K, les cônes figurent les alternatives et les expansions associées, certaines révisent en profondeur l'identité de l'objet ; les connaissances s'étendent soit par production de nouvelles connaissances en lien avec la progression du raisonnement de conception soit en établissant des liens avec des sources de connaissances externes à l'organisation (que celles-ci préexistent ou que leur production soit là aussi stimulée par l'effort de conception). 
Armés de ce formalisme nous pourrons distinguer ce qui dans les raisonnements poursuivis relève de l'un ou l'autre régime de conception mais aussi repérer les liens et les interdépendances entre la Conception innovante et la Conception Réglée.

\section{3 - Gustave Eiffel, l'organisation de la conception innovante, l'exploration de nouveaux potentiels de valeur et la régénération de la conception réglée}

Les expériences menées par Gustave Eiffel dans les établissements qui portent son nom s'imposent comme un cas particulièrement intéressant pour explorer cette question des relations entre conception réglée et conception innovante, entre Bureau d'Etudes et innovation. Gustave Eiffel se qualifie lui-même d'ingénieur et constructeur, mais c'est aussi grand entrepreneur et un organisateur hors pair. Il n'a cessé d'innover tout au long de sa carrière (architecture novatrice du pont sur le Douro au Portugal, nouveau principe de lancement des tabliers, observatoire de Nice, structure métallique de la statue de la liberté,... Et pourtant c'est le même Gustave Eiffel qui organise dès 1877 un bureau d'études exemplaire qui remportant de très nombreux appels d'offres va répandre de part le monde des ouvrages d'art partageant de nombreuses règles de conceptions et un savoir faire commun.

Ces multiples facettes, loin d'être contradictoires, illustrent de notre point de vue un véritable talent de concepteur et d'organisateur de la conception. En montrant comment Gustave Eiffel a cherché en permanence à articuler conception réglée et conception innovante, en épaulant l'une par l'autre, on peut recomposer une figure plus originale et restaurer l'effort d'organisation poursuivi.

Ce choix s'explique aussi par l'importance des sources facilement accessibles. Nous disposons en effet de plusieurs écrits de Gustave Eiffel lui-même [18] [19] [18] [20] $[21,22]$, de nombreuses biographies bien documentées sur lesquelles nous avons pu nous appuyer [23] [24] [25] [26] [27] [28] et des éléments sur la construction métallique et les entreprises concernées [29] [30].

Nous ne retiendrons dans le cadre de cet article que deux exemples significatifs : la tour de 300 mètres et les ponts portatifs économiques.

Conception Réglée des ponts métalliques des Ets G. Eiffel

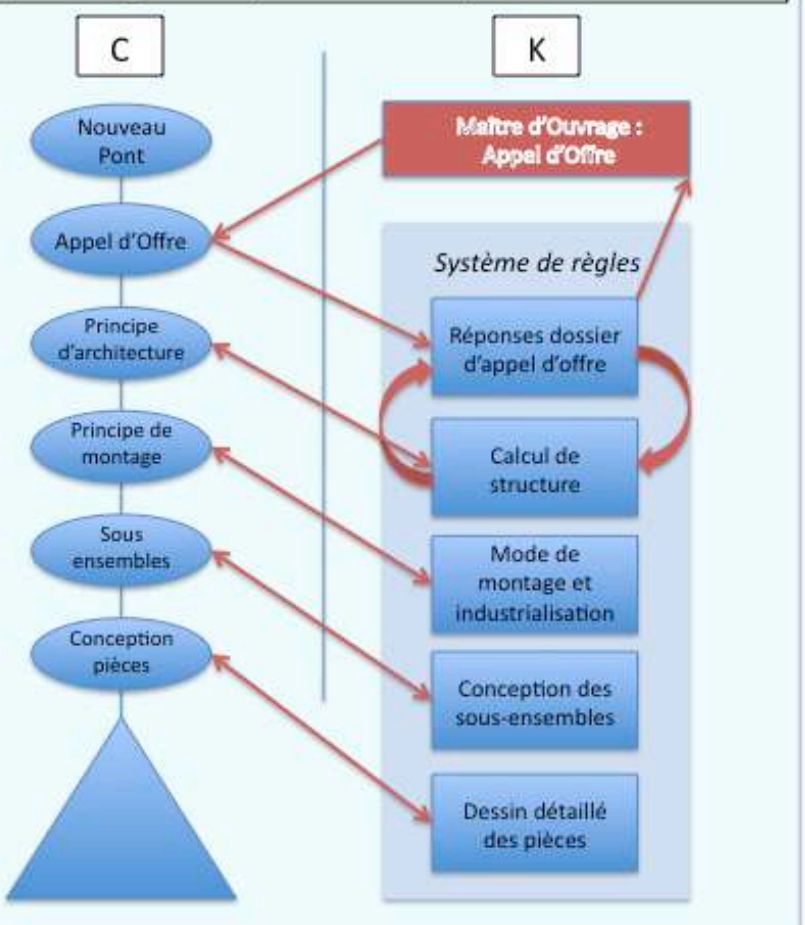

\section{La conception de la tour de 300} mètres

\section{- Les acquis de la conception réglée}

En 1884, au début de l'histoire de ce qui deviendra plus tard la tour Eiffel, Gustave Eiffel a déjà une grande réputation et une vaste expérience des constructions métalliques en particulier des ponts et viaducs pour le chemin de fer. Il dirige une entreprise à son nom et dont les ateliers sont installés à Levallois Perret. Elle abrite un important Bureau d'Etudes qui joue un rôle déterminant dans le succès de 
l'entreprise : il prépare les dossiers de réponse aux appels d'offre, calcule les structures, dessine les pièces et prévoit leur assemblage, conçoit des dispositifs de montage qui assureront la bonne marche du chantier et la réalisation de l'ouvrage. Il s'appuie pour ce faire sur un système de règles de conception qui a été beaucoup étoffé au cours des projets successifs. La figure 3 modélise ce raisonnement dans notre formalisme, il suit typiquement la logique de la figure $2 \mathrm{a}$.

\section{- Un exercice de conception innovante qui dépasse le cadre de la conception réglée}

Ce n'est pas Gustave Eiffel qui aura le premier l'idée de la «tour de 300 mètres » mais Maurice Koechlin et Emile Nouguier, les patrons du Bureau d'Etude et de calcul. Cette idée hantait l'esprit des ingénieurs de l'époque. Clarkes et Reeves imaginaient de construire à Philadelphie pour l'exposition de 1876, une tour de plus de mille pieds composée d'un cylindre en fer de 9 mètres de diamètre maintenu par des haubans. Bourdais n'avait-il pas projeté en 1881 une «tour soleil », un gigantesque phare pour éclairer tout Paris à l'électricité ?

La première esquisse de Koechlin et Nouguier de 1884 est un pylône, une structure métallique analogue à celles qu'ils ont l'habitude de concevoir comme piles de pont, simplement beaucoup plus haute. La tour de 300 mètres ne serait alors qu'un point extrême du cône de la conception réglée de la figure 3 ? Oui, mais inatteignable en suivant le chemin de la conception réglée habituelle. Car en fait le système de règles en vigueur ne permet pas de la concevoir, aucune des étapes du raisonnement pré-établi ne tient et de nombreuses règles font défaut. Tout l'effort de conception va précisément consister à les reconcevoir, à régénérer le système de règles et à en repenser la cohérence.

Au départ Gustave Eiffel n'est pas convaincu par le projet il laisse pourtant Koechlin et Nouguier poursuivre leur conception. Certes la tour sera comme un pylône, mais sa forme doit être reconçue. La tour, si elle ne porte aucune charge, lutte contre le vent et son propre poids, d'où sa forme originale, en chainette, établit par calcul. Ces méthodes de calcul sont connues et maîtrisées par la conception réglée mais elles sont utilisée ici dans une configuration très différentes des situations habituelles. Pour rendre leur tour acceptable dans la ville, ils demandent à Stephen Sauvestre l'architecte avec lequel la société Eiffel collabore habituellement, d'«habiller» le pylône. Celui-ci accroît l'impression de stabilité en dessinant une arche monumentale, il prévoit une grande salle vitrée au premier étage et décore les quatre faces.

Mais il manque toujours un élément essentiel, il n'y a pas d'appel d'offre pour un tel ouvrage. Gustave Eiffel, convaincu de l'intérêt du projet par les progrès de la conception, se lance alors dans une vaste opération de lobbying. Il verrait bien sa fameuse tour devenir le fleuron de l'exposition universelle qui doit être organisée à Paris en 1889 pour commémorer le centenaire de la révolution, effacer les souvenirs de la défaite de 70, faire valoir l'industrie française et relancer l'économie. Il convainc Edouard Lockroy, ministre du commerce et de l'industrie, chargé de l'organisation de l'exposition de faire figurer dans l'appel d'offre une tour de $300 \mathrm{~m}$ de hauteur et $125 \mathrm{~m}$ de côté sur le Champ de Mars où doit se tenir l'exposition.

Le projet ne va pas sans susciter de vives polémiques. La protestation des artistes signée de Charles Gounod, Guy de Maupassant, Leconte de Lisle et bien d'autres est combattue 
par Gustave Eiffel qui défend «la beauté propre de la tour ». La légitimité, acquise à l'époque, quand il s'agit d'un viaduc de chemin de fer doit ici être conçue.

Le montage économique et financier demande aussi un effort de conception original. Il ne prend pas la forme d'un appel d'offre comme dans les grands projets d'ouvrage d'art de l'époque. Une convention tripartite inédite lie l'Etat, la ville de Paris et Gustave Eiffel. Celui-ci assumera les coûts et les risques de la construction contre une subvention de $20 \%$ du montant estimé du coût de la construction, d'un droit d'exploitation à ses risques et périls pendant l'exposition et d'un usufruit de 20 ans sur les recettes de l'exploitation transférée à la ville après l'exposition en 1889. Pour financer le projet Gustave Eiffel avec le concours de trois banques constitue alors une société pour l'exploitation de la tour Eiffel. Gustave Eiffel apporte ses droits d'exploitations en contrepartie d'un certain nombre d'action et du financement du montant prévu des travaux. Les actions émises rencontrent un grand succès auprès du public, les souscripteurs étant attirés par la perspective de bénéfices liés à la fréquentation espérée du monument.

Ainsi la conception de la tour de $300 \mathrm{~m}$ s'écartait sensiblement de la conception réglée et supposait un intense effort de conception innovante mobilisant de nombreux acteurs inhabituels et introduisant plusieurs dimensions nouvelles dans la conception. 


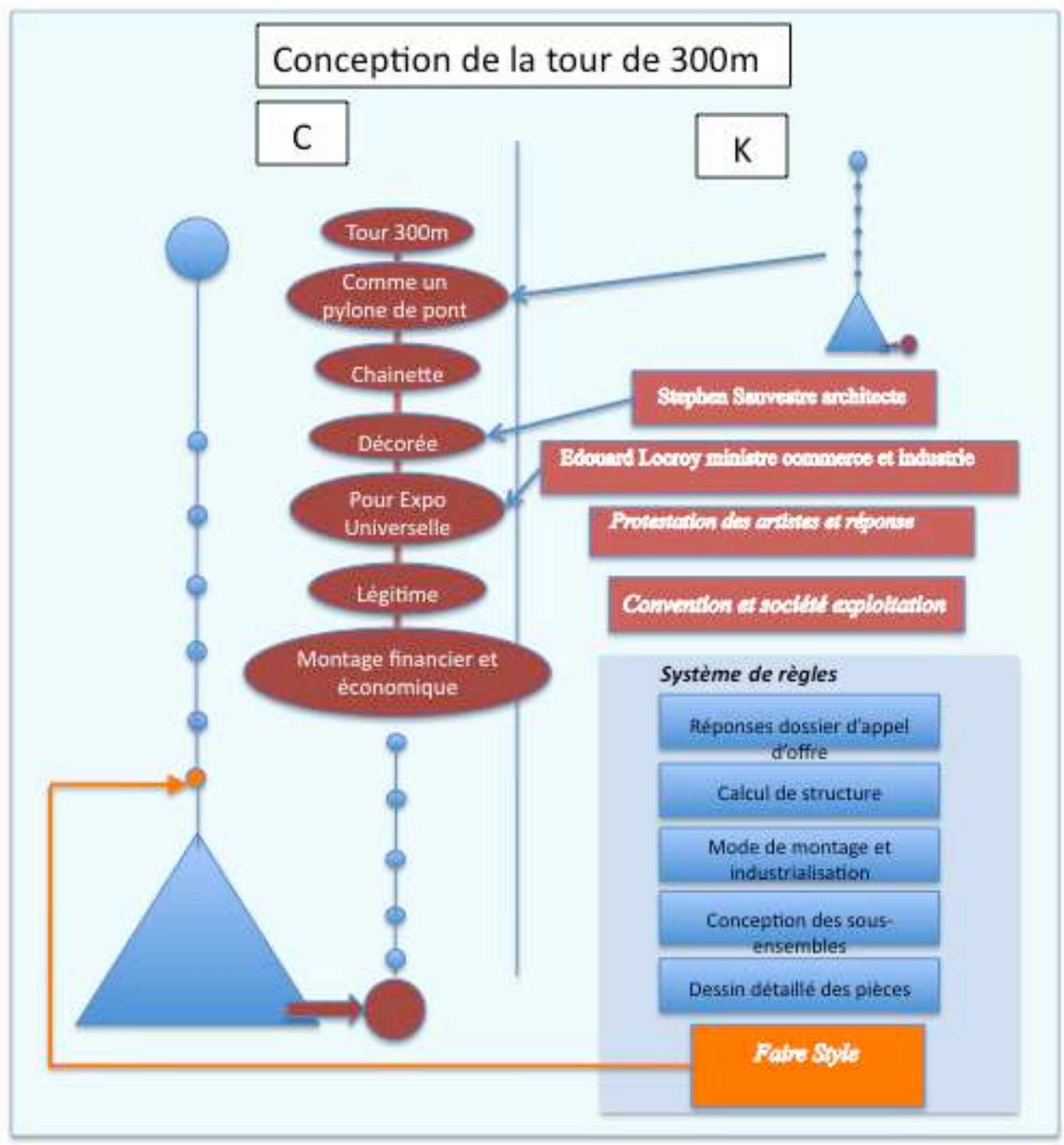

\section{- La conception innovante de la tour Eiffel mobilise les acquis de la conception réglée}

Mais à l'inverse la conception de la tour a très largement bénéficié de la conception réglée, de ses expériences et de son organisation sans lesquels elle n'aurait pas été possible. Donnons en quelques exemples :

Elle emprunte d'abord aux principes mêmes de l'architecture métallique développés et pratiqués par l'entreprise Eiffel : constituer une ossature en treillis par lequel passent les efforts, ce qui permet le calcul et le dimensionnement de la structure.

L'idée des vérins qui permettent d'assurer le réglage des arbalétriers au moment de la jonction de la plateforme du premier étage et de garantir son horizontalité, indispensable à la construction des étages supérieurs, provient de l'expérience du pont 
sur le Douro au Portugal. Des dispositifs de réglage astucieux et novateurs avaient seuls permis la jonction entre les deux arches lancées depuis les rives distantes de plus de 160 $\mathrm{m}$. Les mêmes règles de conception ont d'ailleurs été utilisés pour le célèbre pont de Garabit.

La préparation minutieuse et la fabrication des pièces en atelier aux cotes les plus précises, le travail sur le chantier se réduisant à des opérations de montage, est pour certains [26] la grande innovation de Gustave Eiffel dans le domaine de la construction métallique. Cette technique appliquée à la tour de 300 mètres fit l'étonnement des contemporains, surpris par le calme du chantier, la rapidité et la régularité de la construction. Dans ce cas l'industrialisation et la préparation sont poussés à leurs limites : toutes les pièces sont fabriquées avec une grande précision à partir de plans détaillés, les trous de fixation sont pré-percés avec une précision de 1/10 de millimètre, les $2 / 3$ des 2,5 millions de rivets sont posés en atelier grâce à une machine spéciale. La conception de la tour aura occupé plus de 40 personnes pendant deux ans au Bureau d'Etudes, ils produiront 1700 dessins d'ensembles, 3629 plans de détail (en demiegrandeur ou échelle 1/5).

\section{- L'effet en retour de la conception innovante sur la conception réglée}

La conception et la réalisation de la tour sont perçues par les contemporains comme un exploit remarquable. Elle devient l'une des attractions majeures de l'exposition universelle et l'un des monuments les plus connus et les plus visités jusqu'à devenir un symbole de Paris dans le monde entier. Mais ce succès est avant tout celui de Gustave Eiffel qui aux yeux de tous devient le «magicien du fer » et de son entreprise qui y gagne une réputation qui ne se démentira pas. La tour Eiffel n'est plus seulement une construction métallique analogue aux viaducs de chemin de fer qui l'ont précédé. Mais d'un véritable monument qui fait style, magnifie et incarne l'époque. La construction métallique y acquiert ses lettres de noblesse.

Ce caractère de monument ne profite pas qu'à la tour il rejaillit sur toutes les constructions métalliques, en particulier celles de Gustave Eiffel, passées et à venir, qui vont se voir parées d'une dimension nouvelle. Elles vont incarner l'époque, partager un style inscrit dans la modernité et bénéficier d'un peu de l'aura de la Tour Eiffel. Cette logique de la parure [31] n'est pas le moindre effet en retour de la conception innovante sur la conception réglée. Faire «style» et «monumental», incarner et glorifier la modernité n'étaient pas des attributs attendus de la conception réglée des ouvrages métalliques jusqu'alors, nous y reviendrons.

Le succès de la tour Eiffel rejaillit aussi sur l'entreprise et lui offre des perspectives importantes de croissance. L'effet de réputation jouera encore longtemps dans le cadre des appels d'offre. Derrière Eiffage n'entend-on pas encore Eiffel ?

\section{2) Les ponts portatifs économiques}

A côté des grands ouvrages d'art la maison Eiffel s'était fait une spécialité des "ponts économiques portatifs ». Composés de pièces standards, facilement transportables, montables et démontables sans compétences particulières, ils ont rencontré un grand succès dans les colonies, les pays en développement ou les campagnes. Ils présentaient de plus l'avantage pour l'entreprise Eiffel de fournir du travail aux ateliers entre deux projets de grande envergure. Pourtant leur genèse et leur conception semblent paradoxales, elles ne relèvent ni d'un Bureau d'études rompu à la conception réglée de 
grands ouvrages d'art, ni d'un innovateur entreprenant. Pourquoi et comment l'entreprise Eiffel a-t-elle étendu son activité à ces produits surprenants ?

Gustave Eiffel nous donne de précieuses indications dans sa « Notice sur les nouveaux ponts portatifs économiques » de 1887 à partir desquelles on peut reconstituer et modéliser le raisonnement suivi dans la figure 4 .

\section{- d'une demande décalée à la valuation d'un concept original d'une famille de pont d'un nouveau type}

Le texte indique d'abord que la question des ponts portatifs n'est pas nouvelle et évoque une première expérience en 1873, en Bolivie. L'entreprise Eiffel est sollicitée pour concevoir et construire des ponts dont les pièces doivent être transportées à dos de lamas. Ces ponts seront des poutres triangulées, composées de pièces rectilignes pesant $70 \mathrm{~kg}$ et assemblées par des boulons pour être "montées sans le secours d'aucun ouvrier d'art».

En 1879, occupé à créer un type de pont pour les armées en campagne en Cochinchine, G. Eiffel s'entretient avec le gouverneur qui lui fait part de son souhait de développer rapidement les voies de communication, pour cela il faudrait des ponts simples, démontables, transportables, d'ouvertures variables afin de franchir facilement les multiples cours d'eaux.

Ces expériences et cette demande apparaissent là encore comme des points extrêmes. Proche du cône des ponts que la conception réglée permet de concevoir mais suffisamment décalés pour être inatteignables en appliquant le système de règles issu des expériences passées sur les ponts pour les chemins de fer (un pont portatif est un oxymore, est-ce encore un pont ?). Jamais un Bureau d'études en conception réglé ni un grand innovateur-entrepreneur n'auraient prêté attention à ces demandes, nous avons là la trace d'un raisonnement de conception innovante. Pourtant Eiffel accepte ces «briefs » décalés proposés par des clients inhabituels.

Ces expériences et cette demande lui révèlent un potentiel de valeur nouveau. La multiplication et la variété des situations d'usage de ces ponts démontables permet d'envisager la création d'un nouveau marché intéressant. Il s'aperçoit aussi à cette occasion que ces ponts qui ne sont plus des ponts forment une nouvelle espèce dont la conception pourrait obéir à un nouveau système de règles. C'est la rencontre de ces deux considérations qui intéresse G. Eiffel : il y a là un potentiel de valeur atteignable par les moyens de la conception réglée. Si l'on peut dégager un modèle objet, « un nouveau type de pont-portatif », et si on peut construire un système de règles de conception associé, alors on aura des "ponts portatifs économiques» car les effets d'économie de la conception réglée joueront à plein et la valeur potentielle en sera grandement augmentée. L'ajout de cet attribut " économique » présente un autre intérêt, il renforce le décalage du concept initial et l'obligation de briser des règles de conception du système de règles habituel.. Il ne considère donc pas ces projets comme des cas isolés, à traiter en dehors de l'organisation. Au contraire c'est le point de départ d'une intense activité de conception innovante qui s'appuie sur la conception réglée et ne perd jamais de vu l'enjeu d'un retour vers la conception réglée.

\section{- L'exploration innovante et la reconstruction d'un nouveau système de règles cohérent avec la nouvelle espèce}


Le texte indique "cette question sous son apparence modeste, présentait de grandes difficultés ». Il évoque les nombreux essais nécessaires pour parvenir à concevoir un type de pont satisfaisant. Ces essais ne résultent pas de la multiplication d'essais erreurs tous azimuts, mais d'un raisonnement structuré.

L'effort de conception innovante consiste à rendre activable par le Bureau d'Etudes le cahier des charges fonctionnel donné par le gouverneur de la Cochinchine. Ainsi pour être simple et portatif le pont sera composé de pièces standard, en petit nombre et légères, la taille variable du pont sera assurée par la standardisation des pièces. La facilité de montage sera obtenue par l'usage de boulons. La facilité de mise en place par la légèreté de l'ensemble de la structure qui permettra de reposer directement sur les rives sans préparation et par un procédé de lançage.

L'adoption des boulons comme mode d'assemblage à la place des rivets utilisés traditionnellement casse une règle de conception et soulève immédiatement une question quand à la rigidité de la structure. Les rivets rendaient solidaires les éléments assemblés, le risque avec les boulons est que les jeux n'occasionnent des déformations et ne fragilisent la structure. Il faut donc établir de nouvelles règles de conception qui vont garantir la rigidité : on jouera simultanément sur des perçages précis et des boulons ajustés à bout conique pour s'introduire facilement et sur le croisement des pièces qui vont encore diminuer les jeux. La légèreté sera obtenue en changeant le matériau, on utilisera de l'acier de préférence au fer (qui permet un chargement double). Alors que l'ensemble des règles de conception de l'entreprise Eiffel était associé à l'usage du fer.

Beaucoup d'efforts et d'ingéniosité sont consacrées à la génération d'une panoplie de pièces standards avec le minimum de pièces différentes, faciles à distinguer et à monter et qui concilient l'ensembles des contraintes à respecter. Cette logique de panoplie et de standardisation est très nouvelle pour la construction métallique. Elles sont dimensionnées en utilisant le calcul des charges, bien maîtrisé par le BE, et en se plaçant dans les configurations de pont les plus défavorables, par exemple portée maximale, puis en vérifiant ces résultats par de nombreux essais. On voit à cette étape du raisonnement comment se noue un dialogue entre l'activité de conception innovante et le système de règles et de savoir établis de la conception réglée. Certaines règles sont cassées et doivent être reconstruites, mais les connaissances accumulées par le BE sont précieuses dans certains cas elles permettent de générer ces nouvelles règles. Dans d'autres cas il faut produire des connaissances entièrement nouvelles.

Après de très nombreux essais ce n'est pas un nouveau pont qui est conçu mais un nouveau type de pont et son système de règles associé qui va permettre au Bureau d'Etudes de poursuivre la conception en retrouvant sa logique de conception réglée.

\section{- De la promotion d'une innovation au déploiement d'une nouvelle conception réglée}

La conception innovante n'est pourtant pas encore totalement terminée. Un ensemble de «spécimen» sont installés en France et en Cochinchine. Ces prototypes vont permettre de faire des essais de tenue à la charge et à la déformation. Très souvent les clients eux mêmes procèdent aux essais. Les établissements Eiffel font une large publicité à ces résultats et les utilisent pour certifier de la qualité et valider l'efficacité. En parallèle la promotion de ces nouveaux ponts portatifs est assurée en les présentant dans des grandes expositions où ils reçoivent « les plus hautes récompenses ». L'afflux 
rapide «de commandes considérables » atteste du succès et devient un élément de promotion.

Pendant ce temps la conception réglée ne reste pas inactive, elle génère rapidement une dizaine de familles de ponts portatifs pour couvrir les situations les plus variées (route, chemin de fer, armée, portées variables,...). Ces familles réutilisent au maximum des pièces déjà existantes mais surtout sont obtenues à partir du nouveau système de règle de conception des ponts portatifs. Elle retrouve sa logique traditionnelle en se donnant pour but d'étendre encore la variété des situations couvertes. Les clients intéressés mais qui ne trouveraient pas dans les familles de pont existants celui qui leur conviendrait sont invités à soumettre ces cas particuliers à l'entreprise. Le but on l'aura compris est de générer une nouvelle famille plus qu'une réponse singulière.

Avec les ponts portatifs économiques on assiste à la création d'une nouvelle espèce. C'est une nouvelle famille de ponts accessibles à la conception réglée, mais il a fallu bâtir un nouveau système de règles qui lui soit adaptée. La structure des bases de règle « réplique » celle traditionnelle des ponts pour chemin de fer mais leur contenu a dû être entièrement refondu et adapté à cette nouvelle classe de situations.

\section{3) Eiffel, entre conception réglée et conception innovante}

Quels enseignements tirer de ces deux exemples?

\section{1) La conception innovante aux côtés du Bureau d’Etude : faire évoluer la conception réglée}

En réponse à notre première question de recherche ils attestent l'existence, à côté de la conception réglée incarnée par le Bureau d'Etudes, d'une seconde forme d'organisation collective de la conception tournée vers la conception innovante.

Si la conception réglée appuyait son raisonnement sur l'utilisation d'un système de règles établies, la conception innovante s'oriente dans des explorations plus ouvertes guidées par des concepts inatteignables avec les seules connaissances disponibles. Mais si, chez Eiffel, la conception innovante se lance dans l'exploration de l'inconnu ce n'est pas dans l'unique but de réaliser une innovation singulière. Au contraire cet effort n'est entrepris et ne prend tout son sens que mis en perspective avec la conception réglée et son évolution. Dans nos deux exemples les objets se transforment, de nouvelles identités et de nouveaux attributs apparaissent, le système de règles est révisé ou étendu.

Autant les formes de raisonnement sont relativement faciles à établir et à suivre autant la description de l'organisation est plus difficile à capturer. Ces organisations de la Conception Innovante ne se décrivent pas facilement dans les termes habituels des organisations, les structures et les procédures. Cette activité collective consiste en un métabolisme [Le Masson 2006]. Elle n'est pas non plus pérenne mais apparaît et disparaît en fonction des besoins. C'est pourquoi elle échappe généralement aux commentateurs et aux chercheurs ou est rabattue sur l'activité d'un acteur emblématique, l'innovateur. En s'appuyant sur la modélisation fine du raisonnement de conception suivi on peut néanmoins dégager quelques caractéristiques : elle suppose du temps, elle s'appuie sur la répétition et des explorations multiples, elle demande des ressources et des investissements spécifiques. Gustave Eiffel n'assume pas seul la conception mais il met en place et organise une logique d'action collective. Il jouera bien 
sûr un rôle de premier plan, mais elle implique aussi bien des acteurs inhabituels en dehors de l'entreprise que les acteurs de l'entreprise et de la conception réglée mais qui agiront dans un cadre et une perspective différente. Dans la conception réglée la logique d'action était guidée par les connaissances (K-driven), en conception innovante c'est les concepts qui vont guider l'exploration (C-driven). Comment s'opère cette exploration? Qu'il s'agisse des premières esquisses de Koechlin et Nouguier pour une tour de $300 \mathrm{~m}$ ou des première tentatives de ponts transportables en Bolivie l'exploration se déploie d'abord dans des espaces modestes, volontairement limités qui non ni l'ampleur ni l'ambition du concept d'ensemble ultérieur. Ces espaces spécifiques que nous avons qualifié de « design space » [7] permettent au concepteur de commencer à travailler et à apprendre sur ce qu'il y a à apprendre. Gustave Eiffel semble laisser à ses concepteurs une certaine liberté pour explorer de tels "design space». Mais assez rapidement il assure un pilotage rapproché et oriente la poursuite des explorations, c'est ce que nous avons appelé le «value management » [7]. L'apprentissage est ainsi guidé et conduit par extension progressive en fonction des apprentissages déjà acquis et des nouvelles questions ou opportunités qu'ils génèrent. La conception d'ensemble progresse également en se construisant pas à pas, les attributs manquants pour la tour sont progressivement identifiés puis ajoutés, la valeur est mieux perçue et évaluée. L'effort de production de connaissances ajusté.

Le potentiel de croissance ouvert par les expériences de conception innovante décrites tiennent autant ou davantage au nouveau potentiel atteignable pour la conception réglée révisée et étendue que celle que l'innovation permet d'atteindre directement.

Mais les enseignements les plus intéressants que l'on peut tirer de ces deux exemples tiennent à l'articulation entre la conception Innovante et la conception réglée.

\section{L’articulation entre conception réglée et conception innovante}

Dans les deux exemples nous assistons au même mouvement : à partir d'une conception réglée et d'un système de règles un effort de conception innovante conduit à proposer une innovation et cette activité de conception innovante conduit à l'évolution du système de règles. Comment le système de règles contribue-t-il au projet innovant et comment la conception innovante contribue-t-elle à l'évolution du système de règles ? Nous examinerons successivement ces deux points.

\section{1- l'activité de conception innovante et ses relations à la conception réglée}

Pour Gustave Eiffel la conception réglée n'est pas universelle, elle est contingente et locale. Il considère qu'elle peut être étendue. Il y a toujours place pour une activité de conception innovante aux marges qui explore de nouveaux potentiels de valeur et une extension ou une régénération du système de règles. Cette activité est organisée suivant des principes dont voici les plus significatifs :

- Repérer et laisser vivre des occasions à la « marge » de la conception réglée : les deux exemples précédents montrent comment Eiffel accepte des briefs décalés qu'ils soient proposés par ses collaborateurs ou des tiers extérieurs à l'entreprise. Mais ces briefs ne sont que légèrement décalés, ils sont presque semblables (la tour est comme un pylône, un pont portatif est encore un pont) mais pas tout à fait. S'ils ne sont pas totalement étrangers à la conception réglée ils sont inatteignables avec le système de règles en vigueur. 
- Traiter ces occasions comme des « concepts » Eiffel ne les pense pas comme des marges, des cas singuliers. Il les réintègre dans un concept plus large, porteur d'un espace de valeur nouveau, qui nécessite un travail de conception innovante, mais en lien étroit avec la conception réglée existante.

- Ces concepts sont des voies de sorties du système de règles, c'est pour cela qu'ils l'intéressent. En rajoutant un attribut qui crée l'étrangeté (pont-portatif, tour de $300 \mathrm{~m}$ ) il oblige à casser le système de règles et à le reconstruire pour trouver un chemin de conception. Mais il ne sait pas à l'avance quelles seront les règles à réviser ou à rajouter. On peut noter que ce procédé s'apparente au «forcing » en mathématique [32] [33] $]^{2}$. Le concept est défini par une suite de propriétés compatibles avec le système de règles plus un élément qui lui est étranger (la nouvelle propriété souhaitée) et qui va obliger à le casser. Pour " forcer» l'existence du concept (donc mener la conception à son terme), après avoir brisé une ou plusieurs règles de conception et il faut reconstruire un système de règles compatible le nouvel objet et grâce auquel il puisse être conçu.

- C'est là qu'intervient la capacité à tirer parti des expériences de la Conception Réglée. Sans le point de départ et l'appui du système de règles de la Conception Réglée il est peu vraisemblable que les tentatives de conception innovantes aient permis d'aboutir aux succès que nous venons d'évoquer.

- Un «forcing » qui révèle de nouvelles valeurs, crée de nouvelles espèces et qui régénère le système de règles: l'expansion réalisée au cours du raisonnement de conception modifie l'identité de l'objet conçu et étend les connaissances disponibles. Elle est de nature très différente dans les deux cas ; la tour ne fait pas qu'emprunter à la pile de pont, elle est monument qui fait style. Elle dote tous les ponts futurs d'un attribut nouveau qui étend l'espace de la conception réglée. Les ponts économiques portatifs sont une nouvelle espèce de pont dont l'émergence n'a été rendue possible que par la mise en place d'un nouveau système de règles cohérent qui réplique le système de règles des ponts traditionnels mais en l'étendant et en le révisant en profondeur pour l'adapter à cette nouvelle situation.

On a donc bien une activité très structurée de conception innovante, mais cette activité est constamment pensée en lien avec la conception réglée en place. La conception innovante n'est pas autonome. Elle ne poursuit pas des objectifs propres, elle vise toujours un effet en retour sur la conception réglée. Son but est de faire vivre, de faire évoluer la conception réglée.

La transformation du la conception réglée suppose donc un passage par la conception innovante qui explore l'inconnu, fait apparaître de nouveaux objets dotés de nouvelles valeurs en produisant de nouvelles connaissances. L'effort de la conception innovante ne s'arrête pas là, il va jusqu'à restaurer un système de règles capable de concevoir une variété de produit au sein des nouvelles lignées.

Figure CR->CI -> DCR

\footnotetext{
2 Le forcing a été introduit en mathématique par Paul Cohen en 1963 pour démontrer l'indépendance de
} l'axiome du choix et des axiomes de Zermelo-Frenkel. 


\section{2) Le renouvellement du système de règles et l'extension du potentiel de valeur}

Les deux exemples précédents de conception innovante conduisent à deux cas très contrastés de renouvellement du système de règles et d'extension du potentiel de valeur.

\section{A) la logique de la parure : mutation de l'espèce par création d'un nouvel attribut}

Dans le cas de la tour de $300 \mathrm{~m}$, on est d'abord fasciné par le décalage conceptuel. Considérer la tour comme un pylône de pont rend possible l'exploit en l'appuyant sur un système de règles établi. La conception réglée est un préalable et une condition la conception innovante. Bien sûr pour mener l'aventure à son terme il faudra étendre les compétences disponibles. Mais ce qui nous semble plus intéressant et plus original, c'est l'effet en retour de l'exploit sur le système de règles. Le caractère monumental de la tour va rejaillir sur toutes les constructions métalliques passées et à venir de Eiffel, elles seront comme des tours en petit. Elles seront "parées » des vertus de la tour et accèderont elles aussi au statut de monument ${ }^{3}$. La tour emprunte le modèle objet de la pile de pont mais elle lui ajoute une dimension nouvelle : « faire style ». Cet attribut supplémentaire allonge la liste des descripteurs du modèle objet et le modèle génératif s'enrichit. Il introduit une capacité de distinction supplémentaire par rapport aux autres ponts et augmente ainsi la valeur des ponts conçus par Eiffel. Il ouvre en même temps un nouvel espace d'expansion conceptuel que ne cesseront d'explorer et d'exploiter les ouvrages d'art ultérieurs. Il est intéressant de remarquer que ce nouvel attribut générique perturbe peu le système de règles établi, il est découplé. L'expansion du côté des connaissances se fait par l'ajout d'une nouvelle base de connaissance presque indépendante et complémentaire.

\section{B) la création d'une nouvelle espèce et mutation du système de règles par effet miroir(transmutation?)}

Avec les ponts économiques portatifs c'est à la naissance d'une nouvelle espèce que l'on assiste. Une nouvelle conception réglée est reconstruite à côté de la première. L'effort de conception innovante produit un nouveau modèle objet qui supportera le développement de cette nouvelle lignée. L'élaboration du nouveau système de règles demande un effort très important. Il est guidé par celui qui préexiste. Sa structure sera la réplique du premier mais le contenu des règles doit être entièrement révisé. C'est à une véritable mutation que les concepteurs doivent procéder.

La régénération de la conception réglée emprunte donc deux chemins différents. L'effort que la Conception Innovante sera plus ou moins radical sur le modèle objet et sur le modèle de compétences. Dans le cas de la tour, l'évolution du modèle objet sera incrémental : on rassemble deux espèces à travers un Modèle objet connu où on a ajouté une variable qui établit la relation ; par contre cela suppose une modification radicale du modèle de compétences puisque des connaissances jusque là étrangère à la conception réglée sont ajoutées («faire style»). Avec les ponts portatifs on a un mouvement

\footnotetext{
${ }^{3}$ Avec les notions de «parure » et de «pointe » Armand Hatchuel [31] propose deux logiques d'expansion dont peut jouer le designer, la première ajoute une propriété nouvelle à l'objet qui augmente sa valeur (dans un double jeu d'incorporation d'une valeur jusque là étrangère et de révélation d'une valeur que l'objet possédait mais qui n'était pas visible), la seconde opère en modifiant plus radicalement l'identité de l'objet.
} 
inverse : le modèle objet est radicalement nouveau, c'est une nouvelle espèce, par contre le système de règles s'il est entièrement reconstruit l'a été à partir de connaissances qui ne sont pas radicalement nouvelles pour le Bureau d'Etudes.

\begin{tabular}{|l|l|l|}
\hline & Evolution du Modèle Objet & $\begin{array}{l}\text { Evolution du Modèle } \\
\text { compétences }\end{array}$ \\
\hline La tour de $300 \mathrm{~m}$ & Incrémental & Radical \\
\hline $\begin{array}{l}\text { Les ponts économiques } \\
\text { portatifs }\end{array}$ & Radical & Incrémental \\
\hline
\end{tabular}

\section{Pouvoir d'expansion : mieux que la Conception Réglée ou la Conception Innovante seuls}

Enfin nous voudrions souligner qu'en matière d'expansion (et donc de potentiel de croissance) la combinaison de la Conception Réglée et de la conception Innovante fait mieux que chacune d'entre elles considérées isolément. Nous retrouvons là encore le double mouvement que nous avons déjà souligné : la CI s'appuie sur la CR et la CR relaie la CI.

Leur combinaison rend atteignable des points qui semblaient inaccessibles. A tous les contemporains une tour de 300 mètres semble un rêve, un projet fou, irréalisable avec les connaissances de l'époque. C'est en établissant un rapport inattendu entre une tour et une construction métallique que la situation de conception se transforme. La distance conceptuelle et la distance sur les connaissances à franchir se réduit. La construction métallique offre simultanément un modèle objet candidat et un système de règles en partie utilisable. Les ponts économiques portatifs appartiennent à une autre catégorie, celles des innovations orphelines. Le projet semblait bien trop modeste et concerné un univers trop éloigné pour retenir l'attention d'un spécialiste de la construction métallique de grands ouvrages d'art, or concevoir une solution sans maîtriser ces connaissances et sans disposer des capacités d'exploration et d'expérimentation était peu probable.

La Conception Réglée prenant le relais de la conception innovante assure la répétition économe de la conception et permet d'atteindre non pas un point isolé mais de couvrir tout un espace de points.

\section{4) Discussion et perspectives}

Gustave Eiffel ne s'est donc pas contenté d'organiser un Bureau d'Etudes sur les bases de la Conception Réglée. Il a porté une attention permanente à sa régénération régulière et a l'extension de son potentiel. Pour cela il a organisé en lien avec la Conception Réglée des efforts répétés de Conception Innovante. Combinant une certaine liberté d'exploration de ses collaborateurs et une implication personnelle importante dans le pilotage et l'orientation de ces aventures.

Nous avons donc avec ces deux exemples une première indication en faveur de nos hypothèses. Pourtant à ce stade plusieurs questions méritent d'être soulevées.

\section{A- N'est-ce pas un cas singulier?}


Eiffel est-il un cas à part ? Cette préoccupation d'étendre et de régénérer la conception réglée par la conception innovante est-elle partagée par les premiers industriels qui ont organisés à la fin du XIX ème siècles les premiers grands Bureaux d'Etudes? Incontestablement répondre à cette question suppose de poursuivre l'effort de recherche engagé.

Pourtant nous pouvons déjà évoquer d'autres cas intéressants qui vont dans le même sens et que faute de place nous ne pourrons pas présenter ici en détail.

La capacité des Baldwin Locomotive Works de Philadelphie [2] à réorganiser régulièrement sa conception réglée en changeant à chaque génération le langage de description des produits, ses relations avec ses clients et les bases de fonctionnement de la conception réglée réinventée à chaque transition, est un des facteurs qui explique que cette firme soit devenue le leader incontesté de la conception et la construction de locomotive aux Etats Unis pendant toute la seconde moitié du XIX ème siècle. Le rôle de la conception innovante assumée par les associés-managers a été de concevoir ces étapes successives.

Un autre exemple est celui de Thomson-Houston puis de General Electric entre 1890 et 1900 [12] [34]. A côté de Bureaux d'Etudes (Engineering De partment) de plus en plus structurés pour soutenir l'effort de commercialisation et de fabrication de nouvelles stations de production et distribution électrique, une organisation spécifique le Model Room, placée sous la responsabilité de Elihu Thomson, a pour vocation d'inventer de nouveaux concepts (passage au courant altératif) ou d'étendre la gamme des produits (rayon $\mathrm{X}$, véhicule électrique,...) qui seront ensuite pris en charge par les autres département de l'entreprise.

Un autre cas particulièrement intéressant est celui d'Edison. Les biographies récentes [35] [36] montrent que derrière la figure de l'innovateur se cache une organisation très sophistiquée. Un examen plus attentif nous apprend qu'il ne s'agit pas d'invention tous azimuts sans Bureaux d'Etudes, Mais qu'en fait il y a plusieurs Bureaux d'Etudes selon les domaines. Par exemple les travaux sur l'électricité conduits à Menlo Park puis West Orange permettent de structurer le système électrique, son architecture et ses composants pour chacun d'entre eux des entreprises avec un Bureau d'Etudes sont mis en place. Avec toutes ces nouvelles entreprises Edison passent des contrats pour développer des produits et qui lui payent sa recherche "en plus ». Edison n'arrête donc pas son activité quand apparaît un Bureau d'Etudes mais seulement quand il a l'impression qu'il n'y a plus que du développement à réaliser par un bureau d'études. C'est en tout cas l'hypothèse que nous pouvons formuler pour expliquer son retrait du domaine de l'électricité (au moment de la création de Général Electric par fusion entre Thomson-Houston et Edison General). Nous montrerons dans un autre papier que le modèle d'organisation mis en place à West Orange résulte en fait de plusieurs tentatives-explorations successives dont l'enjeu pour Edison était de construire des formes d'organisation collectives favorisant à la fois l'exploration de nouveaux champs d'innovation et le développement de nouveaux business et industries.

Un autre argument tient au contexte de l'époque: changement rapide de la société (urbanisation, montée du travail de bureau,...), multiplication des nouvelles technologies, apparition de nouveaux secteurs industriels et de nouveaux marchés, inventions organisationnelles et de nouvelles formes d'association. La régénération et 
l'extension des Bureaux d'Etudes qui s'organisent et se structurent à cette époque ne pouvaient manquer d'être confrontés à cette question.

Le Bureau d'Etudes serait donc né plus riche et se serait ensuite appauvri. On aurait eu de multiples formes et tentatives qui mêlaient étroitement Conception Innovante et Conception Réglée. L'idée d'une genèse du Bureau d'Etudes seul, visant la routinisation pour la routinisation nous semble être un trompe l'œil. Le Bureau d'Etudes naîtrait toujours d'un effort de conception Innovante et le maintien d'un dialogue avec la conception innovante serait une condition de sa survie.

\section{B- pourquoi cette histoire a-t-elle été oubliée ?}

Surgit alors une question: pourquoi une telle amnésie? L'absence d'une doctrine explicite et d'un langage de description ont fortement joué. On manquait des outils intellectuels pour penser l'expansion. Il n'y a par exemple pas eu de formation associé à ces pratiques. Le modèle de gouvernance associé à ce double modèle n'était pas non plus très simple à expliciter voir à partager. Il est souvent le fait des fondateurs qui ont eu à organiser les deux types d'organisation et leurs interactions.

La routinisation du « réglé » était plus simple que celle de l'innovant et a pu apparaître comme suffisante : la compétition sur des marchés stabilisés avec un dominant design bien installé s'accommode d'une expansion basée sur le potentiel des règles.

\section{C- intérêt pour les débats contemporains}

L'intérêt de cette double nature initiale du Bureau d'Etudes ne nous semble pas qu'historique. Une histoire enrichit de la genèse des Bureaux d'Etudes peut enrichir les débats contemporains sur les formes d'organisations qui permettent d'augmenter les capacités d'innovation des entreprises.

Le cadre théorique que nous avons proposé et testé dans cet article devrait permettre à travers de prochaines recherches de faire progresser la connaissance sur ces questions.

Biblio

[1] David F. Noble, America by design: science, technology and the rise of corporate capitalism (New York: Oxford University Press, 1979).

[2] John K. Brown, The Baldwin Locomotive Works 1831-1915 : a Study in American Industrial Practice (Baltimore and London: The John Hopkins University Press, 1995).

[3] Wolfgang König, Künstler und Strichezieher. Konstruktions- und Technikkulturen im deutschen, britischen, amerikanischen und französischen Maschinenbau zwischen 1850 und 1930, vol. 1287 (Frankfurt am Main: Suhrkamp Verlag, 1999).

[4] Wolfgang König, "Design and Engineering," in The Oxford Handbook of Business History ed. Geoffrey Jones and Johnathan Zeitlin (New York: Oxford University Press, 2007), 374-395. 
[5] François (Ed.) Caron, "Entreprise et entrepreneurs XIX-XXème siècles," in Congrès de l'association française des Historiens Economistes (mars 1980) (Paris: Presse de l'Université de Paris Sorbonne, 1983), 387.

[6] François Caron, Les deux révolutions industrielles du XXe siècle (Paris: Albin Michel, 1997).

[7] Pascal Le Masson, Benoit Weil, and Armand Hatchuel, Les processus d'innovation : conception innovante et croissance des entreprises (Paris: Hermes, 2006).

[8] Hélène Pasquier, "Acteurs, stratégies et lieux de Recherche et développement dans l'industrie horlogère Suisse, 1900-1970," Entreprises et Histoire, no. 52 (2008): 76-84.

[9] M.H. Henderson and B.K. Clark, "Architectural innovation : the reconfiguration of existing product technologies and the failure of established firms," Administrative Science Quaterly 35, no. sept-30 (1990): ?

[10] Clayton M. Christensen, The Innovator's Dilemna: When New Technologies Cause Great Firms to Fail (Boston, Massachusetts: Harvard Business School Press, 1997).

[11] D. Leonard-Barton, Wellsprings of knowledge : building and sustaining the sources of innovation, ed. Harvard Business School Press (Boston: 1995).

[12] W. Bernard Carlson, Innovation as a Social Process: Elihu Thomson and the Rise of General Electric (Cambridge: Cambridge University Press, 1991).

[13] Thomas P. Hughes, Networks of Power, Electrification in Western Society, 1880-1930 (Baltimore \& London: The John Hopkins University Press, 1983).

[14] James E. Brittain, Alexanderson, Pioneer in American Electrical Engineering, ed. Merrit Roe Smith, John Hopkins Studies in the History of Technology (Baltimore: The John Hopkins University Press, 1992).

[15] M.L. Tushman and P. Anderson, Managing Strategic Innovation and Change, a collection of readings (New York: Oxford University Press, 1997).

[16] Armand Hatchuel and Benoit Weil, "A new approach of innovative Design: an introduction to C-K theory," in ICED'03 (Stockholm, Sweden: 2003), 14.

[17] Armand Hatchuel and Benoit Weil, "C-K design theory: an advanced formulation," Research in Engineering Design 19, no. 4 (2009): 181-192.

[18] Gustave Eiffel, "Les travaux de fondation de la tour de 300 mètres," Mémoires de la société des Ingénieurs Civils de France (1887).

[19] Gusatve Eiffel, Nouveaux ponts portatifs économiques système Eiffel, Br. s.g.d.g., applicables au service des chemins vicinaux et ruraux, à celui ds des armées en campagnes, aux chemins de fer à voie étroite et aux routes coloniales. (Levallois-Perret: G. Eiffel, ingénieur et constructeur, 1887).

[20] Gusatve Eiffel, "Les grandes constructions métalliques,” (1887).

[21] Gusatve Eiffel, "Communication sur les travaux de la tour de 300 mètres," (1888).

[22] Gusatve Eiffel, Contamin, and Fouquet, "Note sur les constructions métalliques," in Congrés international des procédés de construction (Paris: Imprimerie et Librairies centrales des chemins de fer, 1889), 25.

[23] Jean Prevost, Eiffel (Paris: 1929).

[24] François Ponceton, Eiffel, le magicien du fer (Paris: 1939).

[25] Maurice Besset, Eiffel 1832-1923 (Paris: 1957).

[26] Bertrand Lemoine, Gustave Eiffel (Paris: Editions Hazan, 1984).

[27] Henri Loyrette, Gustave Eiffel (Paris: Payot, 1986).

[28] Michel Lyonnet du Moutier, L'aventure de la tour Eiffel : Réalisation et financement (Paris: Publications de la Sorbonne, 2009).

[29] Bertrand Lemoine, L'architecture du fer, france XIXème siècle (Seyssel: Champ Vallon, 1986). 
[30] Gilbert Cordier, "A propos de l'œuvre de Gustave Eiffel. Documentation et réflexion sur les circonstances et les méthodes de la construction métallique au XIXe siècle," (Paris: Ministère de la Culture et de l'Environnement, 1977).

[31] Armand Hatchuel, "Quelle analytique de la conception ? Parure et pointe en design.," Le Design, essais sur des théories et des pratiques (2006): 13.

[32] A. Hatchuel, "Mathématiques et conception : essai sur le forcing en théorie des ensembles," in Les nouveaux régimes de conception, ed. A. Hatchuel and B. Weil (Paris: Vuibert, 2008), 115-132.

[33] A. Hatchuel and B. Weil, "Design as Forcing: deepening the foundations of C-K theory" in ICED07 (Paris: 2007).

[34] Harold C. Passer, The Electrical Manufacturers 1875-1900, A Study in Competition, Entrepreneurship, Technical Change, and Economic Growth (New York: Arno Press, 1972).

[35] Paul Israel, Edison: a Life of Invention (New York: John Wiley \& Sons, 1998).

[36] Andre Millard, Edison and the business of innovation (Baltimore and London: The John Hopkins University Press, 1990). 